\title{
INTERFACE DISTURBANCES CAUSED BY PLANE ELASTIC PULSES
}

\author{
MARIA COMNINOU \\ Dept. Mechanical Encineering and Applied Mechanics \\ University of Michigan, Ann Arbor, MI 48109
}

\section{ABSTRACT}

In this article the work of Comninou, Dundurs and co-authors on the subject of interface disturbances caused by plane elastic pulses is brlefly reviewed. Some paradoxical aspects occurring at high coefficients of friction are pointed out. Some new results are also presented.

\section{Introduction}

If two elastic solids are perfectly bonded, the interface is described as bilateral because it can transmit tensile tractions, and the solids neither separate nor slip unless the bond is broken. If there is no bond, the contact interface between the solids caunot transmit tensile tractions, and the shearing tractions that can be sustained are limited by the available friction. In this case, the solids can easily separate and slip, the boundary conditions contain inequalities, and the interface is described as unilateral. The interaction of elastic waves with a unilateral interface has only recently come under study.

In addition to the work of the author and her collaborators [1-11] described in the next sections, the work of $\mathrm{R}$. K. Miller in the modelling of various bonding conditions by generalized friction laws must be mentioned [12-14]. The case of viscous loose bonding has been considered by Murty $[15,16]$ and problems connected with moving punches and interface fracture are the subject of ongoing investigation by L. M. Brock $[17,18]$.

\section{Problem Description}

The first attempt to understand the phenonena involved when an incident wave strikes a unilateral interface involved a number of idealizations $[1,2]$. The two solids were half planes held together by applied uniform pressure, the incident wave was plane harmonic and the interface was assumed frictionless. Under these conditions the disturbance caused at the interface is in the form of localized periodic separation zones propagating with constant speed. In this article more attention is paid to the problems in which some of the assumptions mentioned are relaxed: the plane wave may have arbitrary form (plane pulse) and the interface can transmit frictional forces, which obey Coulomb's law of friction. The coefficients of static and kinetic friction are assumed equal, $f$. The solids are compressed and sheared at infinity by the uniform applied tractions $\mathrm{p}^{\infty}$ and $\mathrm{q}^{\infty}$, respectively. The longitudinal and shear wave speeds, are denoted by $c_{L}$ and $c_{T}$, and bars are used to denote the quantities related to the upper solid. The pulse strikes the interface at an angle of incidence $\theta_{0}$ measured from the normal to the interface and comes from the side of the lower solid.

Before the wave strikes the interface, the latter is in a state of stick. An incident wave of sufficient amplitude causes a disturbance in the form of slip and separation zones propagating with velocity

$$
v=c_{0} / \sin \theta_{0}
$$

where $c$ is the speed of the incident wave. Depending on the magnitude of $\mathrm{v}$ we distinguish two cases:

Case I. $v$ is supersonic with respect to both solids, $\left(v>c_{L}, v>\bar{c}_{L}\right)$. None of the reflected or refracted waves becomes a surface wave. The solutions can be obtained algebraically 
in closed Eorn.

Case II. $v$ is not supersonic. At least one of the reflected or refracted waves becomes a surface wave. The formulation leads to singular integral equations and the solution is obtained by quadrature.

\section{Frictional vs Frictionless Interface}

For a frictionless interface the discurbance involves only separation $[1,2,6,7]$. In Case I, the gaps open smoothly at the leading edges of the zones of separation, but close with discontinuous slopes at the trailing edges. Accordingly, the interface normal tracLiou has jump discontinuities at the trailing edges. In Case II, the behavior of the normal tractions and the slope of the gaps at the trailing edges depends on $v$ and the elastic moduli. For most ranges of $v$, there is a singularity weaker than square root at the trailing edge. Bounded behavior (vanishing normal traction and slope of gap at the trafling edge) occurs for $\vec{c}_{p}>v>c_{1}$. It may also occur for $c_{T}<v<c_{L}, v<\vec{c}_{p}$ depending on the ratio of the shear moduli $\bar{\mu} / \mu$. Herre $\bar{c}_{R}$ denotes the Rayleigh wave speed ${ }^{R}$ of the upper solid. More details can be found in [2].

When friction is considered, the disturbance generally involves a combination of slip and separation zones and the boundary conditions are coupled $[9,10]$. For identical materials the effect of separation can be decoupled and treated separately as in the frictionless case. The normal tractions thus obtained are used as input in Coulomb's frictional slip. For identical materials Case II arises only when $c_{0}=c_{T}$ and $c_{T}<V<c_{L}$ (transonic case), $[11]$.

\section{Formulation}

The boundary conditions are written in a coordinate system moving with the disturbance. We introduce a dimensionless moving coordinate

$$
\eta=k_{0}\left(x_{1} \sin \theta_{0}-c_{0} t\right)
$$

where $x_{1}$ is the cartesian coordinate along the interface and $k_{0}$ represents either the wave number for periodic pulses or the inverse of a characteristic pulse length for pulses of abritrary form. The tractions corresponding to a bonded interface (bilateral solution) can be obtained routinely if tediously [19] and are of the form

$$
\begin{aligned}
& N_{0}(n)=-p^{\infty}+F_{1}(n) \\
& s_{0}(n)=q^{\infty}+F_{2}(n)
\end{aligned}
$$

where $F_{1}(n)$ and $F_{2}(\eta)$ are functions determined by the complex transmission and reflection coeffictents and the pulse shape. In Case $I$, the reflected and refracted pulses retain the shape of the incident pulse. In Case II and for identical materials the normal and shear tractions, but not the individual reflected and transmitted pulses, retain the original pulse shape. Under these circumstances, (3) and (4) simplify to

$$
\begin{aligned}
& N_{0}(n)=-p^{\infty}+A_{0} F(n) \\
& S_{0}(n)=q^{\infty}+B_{0} F(n)
\end{aligned}
$$

where $A_{0}$ and $B_{o}$ are constants and $F(n)$ is the normalized stress pulse shape.

The solution to the unilateral problem, i.e. the problem involving boundary conditions appropriate for slip and separation, is obtained by adding to the bilateral solution a corrective solution. In Case $I$, the corrective solution can be constructed by Fourier series [1,3], by distribution of moving interface dislocations [6] or by distribution of moving point-forces at the interface [9]. In Case II, the corrective solution is conveniently obtained by using moving dislocation distributions $[7,11]$ or Fourier series [2]. The use of Fourier series is appropriate to harmonic waves.

\section{Discussion of Case I (Supersonic)}

In this case the results depend on three parameters $\lambda_{1}, \lambda_{2}, \lambda_{3}$ involving the elastic constants and the angle of incidence $[3,9]$. The parameters $\lambda_{2}^{2}$ and $\lambda_{3}$ are always positive, but $\lambda_{1}$ may be of either algebraic sign. Two possibilities arise

$$
\text { (a) } f<\lambda_{2} /\left|\lambda_{1}\right|
$$

Then a unique solution satisfying all the physical inequalities of the problem is obtained. The transitions from stick to slip and from slip to separation are smooth, 1.e. 
the tangential shift and the gap have continuous derivatives at the leading edges of the slip and separation zones, respectively. A direct transition from separation to stick is possible at the trailing edge, but not reversely at the leading edge.

$$
\text { (b) } \mathrm{f}>\lambda_{2} /\left|\lambda_{1}\right|
$$

This case corresponds to high coefficients of friction, but it can be realized for materials with significantly differing elastic moduli and an angle of incidence such that the largest of the reflection or refraction angles is close to $\pi / 2$. The solution is not unique in the sense that the leading edge of the disturbance cannot be uniquely defined, although it must lie within a restricted range. It is possible to obtain a unique solution by imposing a further condition. For instance, one may require that the gap opens smoothly, or that stick, once established, prevails until it becomes inadmissible. Another peculiarity which occurs when the applied shear lies in the range - fpo $<q^{\infty}<-\left(\lambda_{2} /\left|\lambda_{1}\right|\right.$ $p^{\infty}$ is that the pulse may cause a gap which remains open to infinity despite the presence of the applied pressure.

It is noted that for identical material, $\lambda_{1}=0$ and (b) is not possible.

\section{Discussion of Case II (Transonic)}

Due to the complexity of case II, only Identical materials have been considered. The incident pulse must then be of $\mathrm{SV}$ type and the speed of the disturbance restricted in the transonic range. Since the solution is obtained in most cases numerically, the shape of the pulse must be specified. A parabolic shape in the range $-1<n<1$ has been chosen $[7,11]$. The coefficient $A_{0}$ and $B_{0}$ in the bilateral tractions (5) and (6) are related by

$$
B_{0}=-A_{0} \cot 2 \theta_{0}
$$

No separation occurs for $p^{\infty}>A$, and the disturbance involves only slip. An example involving both separation and slip is considered in Fig. $1\left(\theta_{0}=35^{\circ}, c_{T} / c_{1}=.5, f=.5\right.$, $\left.A_{0}>0\right)$. This Eigure depicts the extents of separation and slip zones for fincreasing $q^{\infty} / A_{0}$ and fixed $p^{\infty} / \mathrm{A}$. The edges of the separation zone are denoted by $\gamma$ and $\delta$ and are independent of $q^{\infty}$ (fixed). The edges of the slip zone are denoted by $\alpha$ and $B$ and vary with $q^{\infty}$. The magnitude of $q^{\infty}$ is restricted by the value

$$
|q|=f p^{\infty}
$$

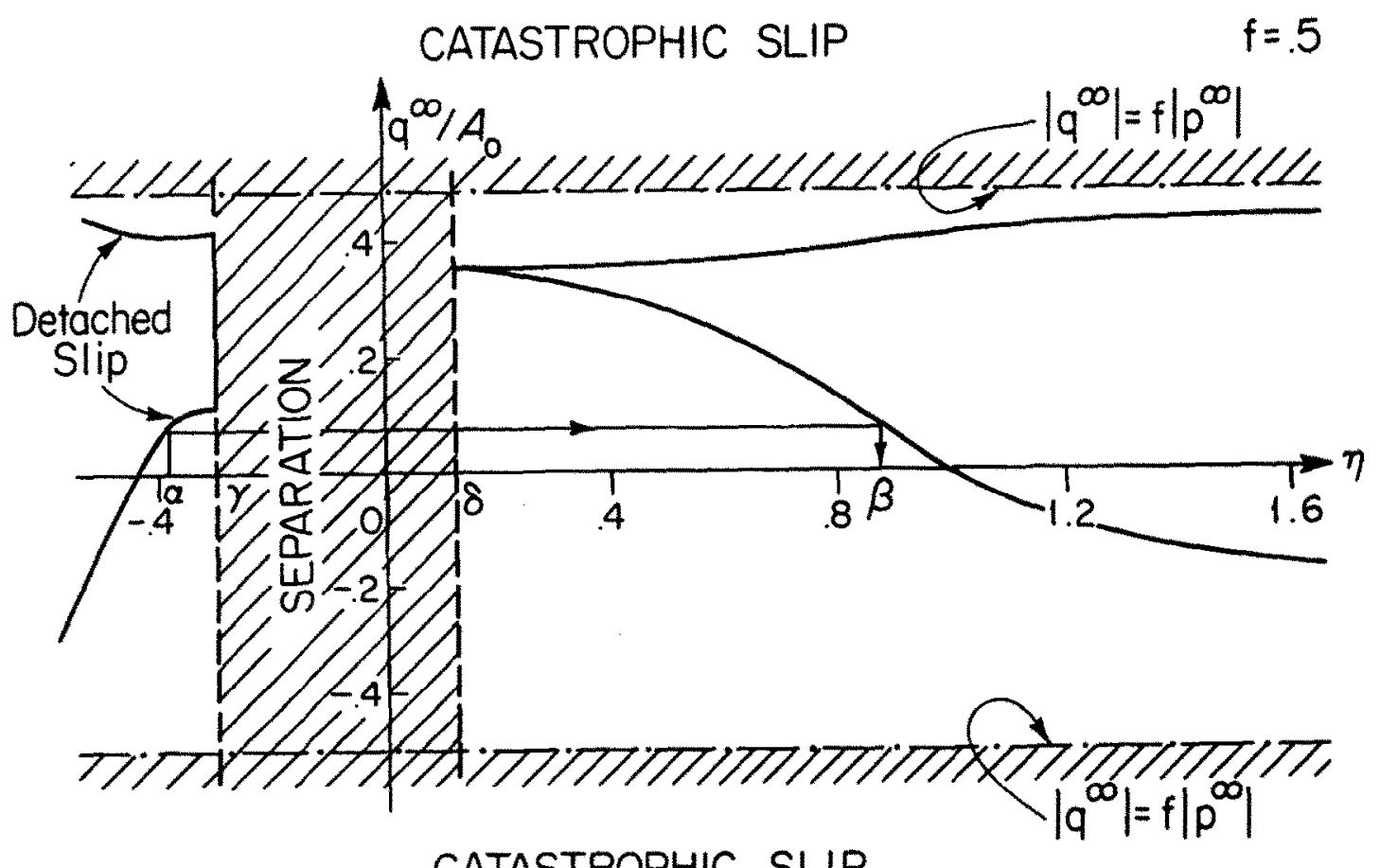

CATASTROPHIC SLIP 
16 which catastrophic slip (over the entire interface) occurs. As q increases from the value $-t p^{\infty}$, the slip, witich is in the direction induced by the pulse, decreases in extent and eventually reaches on the left the trailing edge of the separation zone at some positive applied shear. As $q^{\infty}$ is further increased, the slip zone shrinks on the right up to the leading edge of the separation zone and then slip in the direction of qo (opposite to the direction of the pulse) occurs first ahead of the separation zone. There is a small range in which slip detached from the trailing edge of the separation zone may occur in the vicinity of the points indicated on the figure. Details and other results may be found in [11].

\section{Acknowledgement}

Support by the National Science Foundation under the grant CME 8019281 is grateful1y acknowledged.

\section{References}

1. Comninou, M. and Dundurs, J., Reflection and Refraction of Elastic Waves Involving Separation, Proc. Roy. Soc. Lond.: A356, 509, 1977.

2. Comninou, M. and Dundurs, J., Singular Reflection and Refraction of Elastic Waves Due to Separation, J. Appl. Mech., 45, 548, 1978.

3. Comninou, M. and Dundurs, J., Interaction of Elastic Waves with a Unilateral Interface, Proc. Ruy. Soc. Lond.: A368, 141, 1979.

4. Chez, E. I., Dundurs, J., Comninou, M., Reflection and Refraction of SH Waves in Presence of Slip and Friction, Bull. Seismol. Soc. Am.: 68, 999, 1978.

5. Comninou, M. Dundurs, J. and Chez, E. L., Total Reflection of SH-Wave, in Presence of Slip and Friction, J. Acoust. Soc. Am: 66, 789, 1979.

6. Dundurs, J. and Comninou, M., Interface Separation Caused by a Plane Elastic Wave of Arbitrary Form, Wave Motion: 1, 17, 1979.

7. Comninou, M., and Dundurs, J., Interface Separation in the Transonic Range Caused by a Plane Stress Pulse, J. Sound Vibr.: 62, 317, 1979.

8. Comninou, M. and Dundurs, J., Interface Slip Caused by an SH Pulse, Int. J. Solids Structures: $16,283,1980$.

9. Comninou, M., Barber, J. R., and Dundurs, J., Disturbance at a Frictional Interface Caused by a Plane Elastic Pulse, J. Appl. Mech: 48, 361, 1982.

10. Barber, J. R., Comninou, M. and Dundurs, J., Contact Transmission of Wave Motion Between Two Solids with an Initial Gap, Int. J. Solids Structures: 18, 775, 1982.

11. Comninou, M., Frictional Slip and Separation in the Transonic Range Caused by a Plane Stress Pulse, J. Sound Vibr., in press.

12. Miller, R. K. The Effects of Boundary Friction on the Propagation of Elastic Waves, Bull. Seismol. Soc. Am.: 68, 987, 1978.

13. Miller, R. K. Reflection, Refraction and Absorption of Elastic Waves at a Frictional Interface: SH Motion, J. Appl. Mech.: 46, 625, 1979.

14. Miller, R. K. and Tran, H. T., Reflection, Refraction and Absorption of Elastic Waves at a Frictional Interface: $P$ and SV Motion. J. App1. Mech.: 48, 155, 1981.

15. Murty, G. S., Reflection, Transmission and Attenuation of Elastic Waves at a Loosely Bonded Interface of Two Half Spaces, Geophys. J. Roy. Astron. Soc.: 44, 389, 1976.

16. Murty, G. S. On the Parametric Model of Loose Bonding of Elastic Half Spaces, J. Acoust. Soc. Am.: 60, $1071,1976$.

17. Brock, L. M., Dynamic Rigid Indentation by Sliding Frictionless Contact, Int. J. Engng. Sci.: 16, 545, 1978.

18. Brock, L. M. Dynamic Analysis of Non-Symetric Problems for Frictionless Indentation and Plane Crack Extension, J. Elasticity: 8, 273, 1978.

19. Brekhoviskikh, L., Waves in Layered Media, New York, Academic Press, 1960. 